\begin{tabular}{|c|l|}
\hline Title & Hydrogel with Cubic-Packed Giant Concentric Domains of Semi-Rigid Polyion Complex \\
\hline Author(s) & Wu, Zi Liang; A rifuzzaman, Md.; Kurokawa, Takay uki; Furukawa, Hidemitsu; Gong, Jian Ping \\
\hline Citation & $\begin{array}{l}\text { Soft Matter, 7(5), 1884-1889 } \\
\text { https://doi.org/10.1039/COSM01139H }\end{array}$ \\
\hline Issue Date & 2011 \\
\hline Doc URL & http://hdl.handle.net/2115/52752 \\
\hline Type & article (author version) \\
\hline File Information & ATTO0036.pdf \\
\hline
\end{tabular}

Instructions for use 


\section{Hydrogel with Cubic-Packed Giant Concentric Domains of Semi-Rigid Polyion Complex}

Zi Liang Wu ${ }^{l}$, Md. Arifuzzaman ${ }^{1}$, Takayuki Kurokawa ${ }^{2,3}$, Hidemitsu Furukawa ${ }^{2, \S}$ and Jian Ping Gong ${ }^{2, *}$

${ }^{1}$ Division of Biological Sciences, Graduate School of Science, Hokkaido University, Sapporo 060-0810, Japan;

${ }^{2}$ Faculty of Advanced Life Science, Graduate School of Science, Hokkaido University, Sapporo 060-0810, Japan;

${ }^{3}$ Creative Research Initiative Sousei, Hokkaido University, Sapporo 001-0021, Japan.

[*] Corresponding author

E-mail: gong@mail.sci.hokudai.ac.jp

[§] Present address: Yamagata University, 4-3-16 Jonan, Yonezawa-shi, Yamagata 992-8510, Japan. 


\begin{abstract}
We report a novel giant oriented structure observed in plate hydrogels synthesized by photo-polymerization of cationic monomers with cross-linker in the presence of a semi-rigid polyanion as dopant. The giant structure, formed via self-assembly of the semi-rigid polyion complex, consists of millimeter-scale concentric cylindrical domains in cubic packing that are sandwiched by two homeotropically aligned outer layers. A universal relationship between the diameter of the cylinders $D$ and the thickness of the swollen gel $T$ is observed, as $D=0.5 T$, regardless the change in the concentrations of the polyanion and precursor cationic monomer. This result permits us to induce giant concentric structure into hydorgels with the tunable cylindrical size.
\end{abstract}

\title{
Introduction
}

Hydrogels, a soft and wet matter consisting of cross-linked polymers and a large amount of water, are recognized as the ideal candidate of soft bio-tissues. However, the conventional hydrogels are usually amorphous, weak and with poor functions. In recent years, biomimetic and bioinspired hydrogels have received increasing attention because of their robust functions, high toughness, and potential applications in tissue engineering. ${ }^{[1-7]}$ For the natural materials, a high degree of sophistication is that the various components self-organize into oriented and hierarchical structures following a well-defined pattern. ${ }^{[1,8,9]}$ Thus, liquid crystalline (LC) molecules and block copolymers are often used to develop self-assembled architectures in physically or chemically cross-linked gels. ${ }^{[3-5,10-13]}$ In our previous work, LC hydrogels were synthesized by copolymerizing acrylic acid with LC 11-(4'-cyanobiphenyloxy) undecyl acrylate; these gels show anisotropic shrinkage as temperature increases. ${ }^{[10,11]}$ Also, Thomas and co-workers created a photonic hydrogel with periodic lamellar 
structure by using a block copolymer. ${ }^{[12]}$ However, the anisotropic structures in hydrogels developed in the former works are generally in a sub-micrometer or micrometer level. Hydrogels with macroscopically anisotropic structures are rarely realized.

In recent years, we focused on the synthesis of a semi-rigid polyanion poly(2,2'-disulfonyl-4,4'-benzidine terephthalamide) (PBDT) and its self-assembling behaviors in aqueous solution and hydrogels. ${ }^{[14-21]}$ We have developed hydrogels with macroscopic anisotropic structure by dialyzing the PBDT aqueous solution into a calcium chloride solution. ${ }^{[18]}$ The diffusion of $\mathrm{Ca}^{2+}$ induces molecular orientation and electrostatic complexation of the negative charged PBDT, and thus the formation of anisotropic structure with size of several centimeters in the physical hydrogel. Furthermore, a hydrogel with millimeter-scale anisotropic domains has been synthesized by the thermal polymerization of a cationic monomer $N$-[3-(N,N-dimethylamino)propyl] acrylamide methyl chloride quarternary (DMAPAA-Q) in the presence of a small amount of PBDT as dopant. ${ }^{[15,16]}$ During the polymerization of the isotropic precursor solution, semi-rigid polyion complex forms and self-assembles into randomly dispersed anisotropic domains.

In this paper, we report the discovery of a novel structure for the above system. Concentric cylinder domains at millimeter-scale in cubic packing are observed in the hydrogels synthesized by the photo-initiated polymerization from the two sides of the reaction cell. In the outer layer, the polyion complexes orient perpendicular to the gel surface, since homeotropic alignment on the glass wall is energetically favorable for the semi-rigid polyion complex. In the inner region, the polyion complexes self-assemble to form well-oriented concentric cylinders with their axes perpendicular to the plate gel surface. Moreover, the diameter of the concentric cylinders can be 
simply tuned by changing the gel thickness.

\section{Experimental}

\section{Synthesis of plate poly(DMAPAA-Q) gels containing PBDT}

PBDT, a water soluble semi-rigid polyanion, was synthesized by an interfacial polycondensation reaction. ${ }^{[22]}$ The weight-average molecular weight, $M \mathrm{w}$, and polydispersity $(M \mathrm{w} / M \mathrm{n})$ of PBDT were $1.8 \times 10^{6} \mathrm{~g} / \mathrm{mol}$ and 1.2 , respectively. The cationic monomer DMAPAA-Q (Kohjin Co. Ltd.) and the radical photo-initiator 2-ketoglutaric acid (Wako Pure Chemical Industries Ltd.) were used as-purchased without further purification. $N, N^{\prime}$-methylenebisacrylamide (MBAA) (Wako Pure Chemical Industries Ltd.) was recrystallized from ethanol and used as a chemical cross-linker. The chemical structures of PBDT and DMAPAA-Q are shown in Scheme 1.

The hydrogels are synthesized by a photo-initiated polymerization at room temperature. PBDT used in the current work has a significantly high $M \mathrm{w}=1.8 \times 10^{6}$ $\mathrm{g} / \mathrm{mol}$, much larger than $8 \times 10^{4} \mathrm{~g} / \mathrm{mol}$ that was used in our previous study. ${ }^{[14,15]}$ Aqueous solutions with a prescribed amount of PBDT, DMAPAA-Q, NaCl, MBAA, and 2-ketoglutaric acid were poured into the reaction cells consisting of a pair of parallel glass plates with $0.2,0.3,0.5,1$ or $2 \mathrm{~mm}$ silicone spacer (Figure 1a). Polymerization was carried out by UV irradiation from two sides of reaction cell for 6 $\mathrm{h}$ under argon atmosphere (Figure $1 \mathrm{~b}$ ). The synthesized plate gels were swelled in a large amount of pure water for a week to reach an equilibrium state. The incorporation of $\mathrm{NaCl}$ during polymerization was to enhance the ionic strength of the solution and thus to suppress the occurrence of phase separation due to polyion complex formation; otherwise the gels synthesized with 1.5 M DMAPAA-Q were turbid and showed weak 
birefringence. ${ }^{[16,19,20]}$

Samples are coded as $\mathrm{QP}-C_{\mathrm{Q}^{-}} C_{\mathrm{P}}$ or $\mathrm{QP}-C_{\mathrm{Q}^{-}} C_{\mathrm{P}}+C_{\mathrm{S}}$ (if $\mathrm{NaCl}$ is incorporated), where $C_{\mathrm{Q}}$ is the monomer concentration in $\mathrm{M}, C_{\mathrm{P}}$ is the PBDT concentration in wt $\%, C_{\mathrm{S}}$ is the $\mathrm{NaCl}$ concentration in $\mathrm{M}$. All the samples are synthesized using a constant concentration of chemical cross-linker $(2 \mathrm{~mol} \%)$ and photo-initiator $(0.15 \mathrm{~mol} \%)$ (relative to monomer concentration).

\section{Polarizing optical microscope (POM) observation}

The ordered structures of swollen gels containing PBDT were observed under POM (Nikon, LV100POL) at room temperature. Images were captured by a digital camera coupled with the microscope. All the samples showed a first order white-gray interference color, and $530 \mathrm{~nm}$ tint plate was used to distinguish the alignment direction of PBDT.

\section{Small angle X-ray scattering (SAXS)}

The swollen gel QP-1.5-1 + 0.5 was cut into a small chip with thickness of $\sim 1 \mathrm{~mm}$ and then covered by two Kapton films. SAXS measurements were performed from the lateral direction of the sample at the BL40B2 of SPring-8, Japan Synchrotron Radiation Research Institute, using an incident X-ray with the wavelength, $\lambda=1.6 \AA$. Scattered X-rays were detected using an imaging plate with a resolution of 0.1 $\mathrm{mm} /$ pixel and a $3250 \mathrm{~mm}$ sample-to-detector distance.

\section{Elemental analysis}

The swollen plate gel QP-1.5-1 + 0.5 was cut into three slices using a thin blade, corresponding to different alignment regions, as schemed in Figure $3 \mathrm{~b}$ and Figure 5a. After freeze-drying, elemental analysis of $\mathrm{C}, \mathrm{H}, \mathrm{N}$, and $\mathrm{S}$ were conducted at the chemical analytical center of Hokkaido University, Japan, using the standard techniques. The swelling degree of the gels was estimated by measuring the mass 
ratio of the sample in its swollen state to its dried state.

\section{Results and discussion}

The poly(DMAPAA-Q) gels synthesized without adding PBDT are amorphous. However, strong birefringence can be observed under polarizing optical microscope $(\mathrm{POM})$ while introducing a small amount of PBDT, e.g. $C_{\mathrm{P}}=1 \mathrm{wt} \%$, much lower than the critical concentration of nematic LC, $C_{\mathrm{LC}}{ }^{*}$ of $2.2 \mathrm{wt} \% .^{[18-21]}$ The birefringence is not homogeneous over the sample, with macroscopic Maltese cross occasionally, indicating the existence of either a radial or a concentric structure by molecular orientation (Figure 2). The interesting phenomenon is that, after immersing the as-prepared gel into pure water, many Maltese crosses appear that do not change with the rotation of the samples, and they array in a well-defined cubic packing (Figure 3a, upper image). This permits us to quantitatively characterize the structure of the Maltese crosses of the gel in the swollen state.

Since the polycation is a flexible polymer with much shorter persistence length, the birefringence of PBDT-containing gel is considered mainly from the oriented PBDT, which is a positive LC, as has been clarified from the birefringent colors of its aqueous solution under shearing. ${ }^{[21,23-26]}$ PBDT shows blue and orange colors if oriented in southwest and northwest directions observed with $530 \mathrm{~nm}$ tint plate, respectively. From the birefringent colors of PBDT-containing hydrogel, one can determine the orientation of PBDT and its polyion complex in the hydrogel; this method was confirmed by small angle X-ray scattering (SAXS) in our previous study. ${ }^{[21]}$ Therefore, the birefringent image observed with tint plate in Figure $3 \mathrm{a}$ (lower image) indicates that cubic-packed, millimeter-scaled concentric structures correspond to the orientation of PBDT molecules or the PBDT complexes along the 
circular direction of the cylinder, as illustrate in Figure 3c (left illustration). ${ }^{[21,26]}$

Then, we observe the structure of the gel from the lateral direction (cross-section of the plate gel) under POM. When the sample is placed at $45^{\circ}$ against the polarizer or analyzer, it shows strong birefringence in the center region as well as in the two surface regions close to the substrates, and between the center region and the surface region, dark lines parallel to the surfaces are observed (Figure 3b, upper image). The sample becomes completely dark when it is placed in parallel to the polarizer or analyzer. These results clearly indicate that the PBDT molecules are either oriented in parallel to or in vertical to the surface. In order to identify the PBDT orientation, we further observe the sample with the $530 \mathrm{~nm}$ tint plate. The green color in the surface regions and orange color in the center region shown in Figure $3 b$ (lower image) indicate that the molecules in the surface regions are oriented in vertical to the surface while those in the center region are in parallel to the surface, as schematically illustrated in Figure 3c (right illustration). So in the two outer regions in contact with the glass substrate, PBDT or its polyion complex homeotropically aligns on the glass substrate with a millimeter scale thickness.

The parallel orientation of the PBDT molecules in the center region and the homeotropic orientation in the surface regions are also confirmed by the SAXS measurements (Figure 4). X-ray was irradiated from the lateral direction of the gel, as shown in Fig. 4a; the scattering points relate to the outer and inner regions of the gel with different molecular alignments. Both scattering patterns are elliptical, despite of very weak, indicating the molecular alignment perpendicular to the long axis of the elliptical shape (Figure $4 \mathrm{~b}$ and Figure 4c). The preferential molecular alignment determined by SAXS is consistent with the POM observation. To be more specific, for example, the elliptical scattering pattern of the inner region has a long axis of the 
ellipsoid in the meridian (Figure 4c), indicating that the average distance between PBDT/polyion complex aligned parallel to the substrate is slightly shorter than that aligned vertical to the substrate. No distinct intensity variation is observed along the circular direction of the scattering plane. The POM micrographs and SAXS measurements suggest that the nematic LC structure with a low order parameter is formed in the gel. The slight diffuse scattering peak in Figure 4 corresponds to an average characteristic spacing of $\sim 10 \mathrm{~nm}$, suggesting the spatial correlation among the semi-rigid polyion complexes formed by the strong electrostatic interaction. ${ }^{\text {[27-29] }}$

The above side view observation in Figure $3 \mathrm{~b}$ indicates that the plate shaped gel has a millimeter scale layered structure in its thickness direction. So we further slice the gel in its thickness direction into 3 pieces of different oriented layers, and observe these slices from the top surface direction (Figure 5a). Both slices of the outer layers show very weak birefringence (Figure $5 \mathrm{c}$ ). On the other hand, the slice of the middle layer exhibits strong birefringence of well-arranged Maltese crosses (Figure 5d), quite similar to that of the original bulk gel (Figure $5 \mathrm{~b}$ ). Thus, we confirm that the concentric giant domains exist in the inner region, as shown in Figure 3c. As a summary, giant concentric cylinders composed of semi-rigid polyion complex are cubically packed in the inner region of the hydrogel, sandwiched by homeotropically aligned surface layers.

When we stack the three slices together, they exhibit the same patterns without any decay in the birefringence in comparison with the original bulk gel, as observed from both the top surface and cross-section directions under POM (Figure 5e). Thus, the formation of cubic-packed cylindrical structure does not come from any internal stress mismatch between the surface layer and the inner layer with different molecular orientations. 
The hydrogels with cubic-packed giant concentric domains can be developed in a large range of $C_{\mathrm{Q}}$ and $C_{\mathrm{P}}$. The diameter of concentric cylinders, $D$, in the swollen gels shows a slight decrease with the increase in $C_{\mathrm{Q}}$ and $C_{\mathrm{P}}$ for the gels synthesized with a constant concentration of cross-linker $(2 \mathrm{~mol} \%)$ and initiator $(0.15 \mathrm{~mol} \%)$, as well as thickness of silicone spacer $(1 \mathrm{~mm})$ (Figure $6 \mathrm{a}$ and $6 \mathrm{~b})$. However, the ratio of $D$ to the thickness of swollen gel, $T$, shows a constant value of $D / T \sim 0.5$, independent of the change in $C_{\mathrm{Q}}$ and $C_{\mathrm{P}}$. These results indicate that the slight change of $D$ in Figure $6 \mathrm{a}$ and $6 \mathrm{~b}$ corresponds to the different swelling ratios because $D / T$ holds the constant value.

In order to elucidate if the ratio of $D / T=0.5$ is universal or not, we systematically changed the thickness of silicone spacer while keeping the other parameters constant. The gels synthesized with different thicknesses of silicone spacer exhibit an identical birefringence pattern, indicative of the formation of cubic-packed concentric cylinders even in a limit confined space of $0.2 \mathrm{~mm}$. $D$ increases with the increment of gel thickness $T$, showing a very good linear relationship, as $D \sim 0.57 T^{1.07}$, over a wide range of $T=0.4-2 \mathrm{~mm}$ (Figure 7) for the samples without addition of simple salt (QP-2-1). When with the addition of $0.5 \mathrm{M} \mathrm{NaCl}$ (QP-1.5-1 + 0.5), we observe a relationship of $D \sim 0.44 T^{1.04}$. Both of them are very close to $D / T=0.5$. This universal relationship between $D$ and $T$ permits us to effectively control the diameter of cubic-packed domains by changing the gel thickness.

What mechanism is responsible for the formation of such well-defined, giant oriented structure in the hydrogels? Polyion complex formation between poly(DMAPAA-Q) and PBDT should be responsible for this structure formation. This is confirmed by the fact that if we use an anionic monomer, 2-acrylamido-2-methylpropanesulfonic acid (AMPS) instead of DMAPAA-Q, both 
the as-prepared and swollen gels exhibit no birefringence due to the absence of polyion complexation. ${ }^{[15,16,19]}$ During the polymerization, polyion complex forms between the polycation and oppositely charged PBDT. ${ }^{[19-21]}$ Primarily, the homeotropic alignment on the glass substrate seems energetically favorable for the semi-rigid polyion complexes, and thus form the oriented structure in the outer region of the gels, where PBDT and its polyion complex align perpendicular to the substrate surface. Similar results are observed in other LC complex system consisting of sulfonic acid and amino groups. ${ }^{[30-32]}$ We should emphasize that the macroscopically homeotropic alignment (at the millimeter scale, $\sim 1 \mathrm{~mm}$ in Figure $3 \mathrm{~b}$ ) of PBDT polyion complex is much more long-range than that found by Ujiie and Mendes, which was at the mesoscopic scale of $\sim 20 \mu \mathrm{m}$.

The polyion complex should have a poor solubility even in the aqueous solution of relatively high ionic strength. Therefore, phase segregation occurs in accompany with the polymerization. Different from the case in solution, the phase-segregated domains are frozen by the chemical cross-linking at a certain size. As has been clarified in a previous study, owing to the significant UV absorbance of PBDT, the polymerization is preferentially initiated on the two surfaces of the sample, therefore, a gradient structure was formed in the PBDT containing gels due to the monomer diffusion from the inner region to the outer region during the polymerization. ${ }^{[21,33,34]}$ This gradient structure is also formed in the present case, as confirmed by the elemental analysis results in Table 1, which shows that the inner region has a relatively low mass ratio of poly(DMAPAA-Q) to PBDT; in turn, it possesses larger mass swelling ratio than the outer region. This result indicates that the polymerization and cross-linking reaction in the inner region of the sample occurs slowly than the two surface regions. The heterogeneity in photo-initiated polymerization might be responsible for the different 
size of the cylinder structure formation. The thicker the reaction cell, the slower the gelation kinetics in the inner region, and this favors the larger structure formation in the inner layer of the sample. The increase in the birefringence and the appearance of the ordered Maltese crosses structure by the swelling process in water possibly results from the enhancement of the polyion interaction due to the diffusion of the small counter ions and added $\mathrm{NaCl}$ out of the gel. ${ }^{[35-37]}$ That is, the ordered structure should be developed during the polymerization and the swelling makes it visible under POM. Further studies are ongoing to elucidate the essential mechanism for the formation of cubic-packed cylinders in the inner region.

\section{Conclusions}

In this work, we have developed a novel hydrogel with millimeter-scale oriented structures by photo-polymerization of a cationic monomer in the presence of semi-rigid polyanion PBDT as dopant. The polyion complexes are formed during the polymerization via the electrostatic interaction that homeotropically align on the glass substrate due to the special interfacial interaction. In the inner region, the polyion complexes self-assemble into cubic-packed concentric cylinders with their axes perpendicular to the gel surface. The diameter of the concentric cylinders universally depends on the thickness of the swollen gel, and the latter is tunable by changing the silicone spacer thickness for polymerization of the plate gel. As far as we know, the hydrogel with millimeter-scale cubic-packed concentric domains, as well as long-range homeotropic alignment of polyion complex, has not been reported before that are expected to find applications in material science and merit developing biomimetic hydrogels with intricate complex structures. 


\section{Acknowledgments}

This research was financially supported by a Grant-in-Aid for Specially Promoted Research (No. 18002002) from the Ministry of Education, Science, Sports and

Culture of Japan. The authors thank Prof. Charles C. Han, Chinese Academy of Science, for his precious discussion. 


\section{References}

(1) C. Sanchez, H. Arribart and M. M. Giraud-Guille, Nat. Mater., 2005, 4, 277.

(2) S. Zhang, Nat. Biotechnol., 2003, 21, 1171.

(3) J. Song, E. Saiz and C. R. Bertozzi, J. Am. Chem. Soc., 2003, 125, 1236.

(4) T. Kato, N. Mizoshita and K. Kishimoto, Angew. Chem., Int. Ed., 2006, 45, 38.

(5) M. Hayakawa, T. Onda, T. Tanaka and K. Tsujii, Langmuir, 1997, 13, 3595.

(6) J. P. Gong, Soft Matter, 2010, 6, 2583.

(7) R. Langer and D. A. Tirrell, Nature, 2004, 428, 487.

(8) K. McGrath and D. S. Kaplan, Protein-Based Materials, Bikhauser, Boston, 1997.

(9) L. P. Gartner and J. L. Hiatt, Color Textbook of Histology, 2nd ed., Saunders, Philadelphia, 2001.

(10)T. Kaneko, K. Yamaoka, J. P. Gong and Y. Osada, Macromolecules, 2000, 33, 412.

(11)T. Kaneko, K. Yamaoka, Y. Osada and J. P. Gong, Macromolecules, 2004, 37, 5385.

(12)Y. Kang, J. J. Walish, T. Gorishnyy and E. L. Thomas, Nat. Mater., 2007, 6, 957.

(13)D. Batra, D. N. T. Hay and M. A. Firestone, Chem. Mater., 2007, 19, 4423.

(14)T. Funaki, T. Kaneko, K. Yamaoka, Y. Ohsedo, J. P. Gong, Y. Osada, Y. Shibasaki and M. Ueda, Langmuir, 2004, 20, 6518.

(15) Y. Shigekura, Y. M. Chen, H. Furukawa, T. Kaneko, D. Kaneko, Y. Osada and J. P. Gong, Adv. Mater., 2005, 17, 2695.

(16) Y. Shigekura, H. Furukawa, W. Yang, Y. M. Chen, D. Kaneko, Y. Osada and J. P. Gong, Macromolecules, 2007, 40, 2477.

(17)W. Yang, H. Furukawa, Y. Shigekura, K. Shikinaka, Y. Osada and J. P. Gong, Macromolecules, 2008, 41, 1791. 
(18)W. Yang, H. Furukawa and J. P. Gong, Adv. Mater., 2008, 20, 4499.

(19)Z. L. Wu, H. Furukawa, W. Yang and J. P. Gong, Adv. Mater., 2009, 21, 4696.

(20)Z. L. Wu, T. Kurokawa, S. M. Liang and J. P. Gong, Macromolecules, 2010, 43, 8202.

(21)Z. L. Wu, T. Kurokawa, S. M. Liang, H. Furukawa and J. P. Gong, J. Am. Chem. Soc., 2010, 132, 10064.

(22)E. J.Vandenberg, W. R. Diveley, L. J. Filar, S. R. Pater, H. G. Barth, J. Polym. Sci., Part A: Polym. Chem., 1989, 27, 3745.

(23)D. Demus, J. Goodby, G. W. Gray, H. W. Spiess and V. Vill, Handbook of Liquid Crystals, Wiley-VCH, Weinheim, 1998.

(24)J. H. Yun, K. Kubayama and T. Ougizawa, Polymer, 2006, 47, 1715.

(25)K. Murata and K. Haraguchi, J. Mater. Chem., 2007, 17, 3385.

(26)S. Haseloh, P. van der Schoot and R. Zentel, Soft Matter, 2010, 6, 4112.

(27) Y. Hayashi, M. Ullner and P. Linse, J. Phys. Chem. B, 2003, 107, 8198.

(28)R. M. Capito, H. S. Azevedo, Y. Velichko, S. A. Mata, and S. I. Stupp, Science, 2008, 319, 1812

(29)S. M. Zhang, M. A. Greenfield, A. Mata, L. C. Palmer, R. Bitton, J. R. Mantei, C. Aparicio, M. O. de la Cruz and S. I. Stupp, Nat. Mater., 2010, 9, 594.

(30)S. Ujiie and K. Iimura, Macromolecules, 1992, 25, 3174.

(31)H. A. Every, L. V. Van der Ham, S. J. Picken and E. Mendes, J. Phys. Chem. B, $2008, \mathbf{1 1 2}, 16403$.

(32)H. A. Every, L. V. Van der Ham, S. J. Picken and E. Mendes, Soft Matter, 2009, 5, 342.

(33) V. Vorflusev and S. Kumar, Science, 1999, 283, 1903.

(34)N. Désilles, L. Lecamp, P. Lebaudy and C. Bunel, Polymer, 2003, 44, 6159. 
(35)K. Wagner, D. Harries, S. May, V. Kahl, J. O. Rädler and A. Ben-Shaul, Langmuir, 2000, 16, 303.

(36)J. Gummel, F. Cousin and F. Boué, J. Am. Chem. Soc., 2007, 129, 5806.

(37)S. Lindhoud, R. de Vries, R. Schweins, M. A. C. Stuart and W. Norde, Soft Matter, 2009, 5, 242. 
Table 1. Element analysis results of swollen gel QP-1.5-1 synthesized in the presence of $0.5 \mathrm{M} \mathrm{NaCl}$. Swelling ratio of the gel (w/w) is also shown in the table.

\begin{tabular}{ccccccc}
\hline Sample No. & ${ }^{a} \%$ & $\mathrm{H} \%$ & $\mathrm{~N} \%$ & $\mathrm{~S} \%$ & $\mathrm{~W}_{\mathrm{Q}} / \mathrm{W}_{\mathrm{P}}{ }^{b}$ & Swelling ratio \\
\hline 1 & 44.3 & 9.6 & 11.5 & 0.19 & 54.6 & 36.1 \\
2 & 43.4 & 9.4 & 11.0 & 0.48 & 20.4 & 58.4 \\
3 & 46.9 & 10.1 & 12.1 & 0.21 & 52.1 & 41.2 \\
\hline
\end{tabular}

${ }^{a}$ Samples from different layers of the swollen gel, as shown in Figure $3 b$ and Figure 5 a.

${ }^{b}$ Mass ratio of poly(DMAPAA-Q) to PBDT estimated from the values of $\mathrm{N}$ and $\mathrm{S}$. The mass ratio in feed was 30.9 .

Table 1. Z. L. Wu et al. 


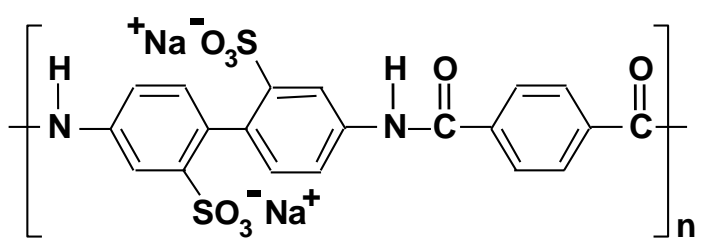

(a) PBDT<smiles>C=CC(=O)NCCCCC</smiles>

(b) DMAPAA-Q

Scheme 1. Chemical structures of (a) semi-rigid polyanion, PBDT, and (b) cationic monomer, DMAPAA-Q.

Scheme 1. Z. L. Wu, et al. 

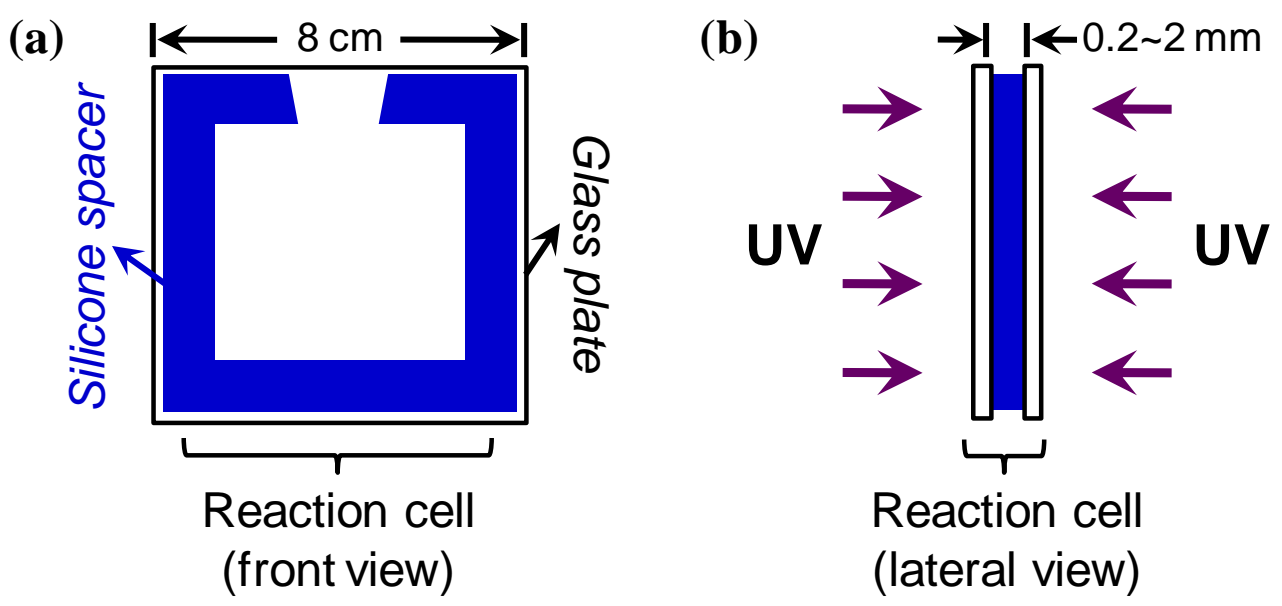

Figure 1. Scheme for the synthesis of plate gel by photo-initiated polymerization. (a) Front view of the reaction cell consisting of a pair of glass plates and a silicone spacer, (b) lateral view of the reaction cell under UV irradiation from the two sides.

Figure 1. Z. L. Wu et al. 


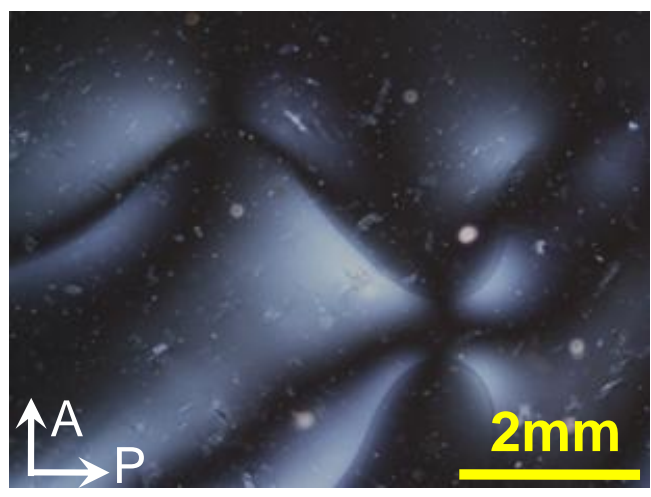

Figure 2. Polarizing optical micrograph of the as-prepared gel QP-1.5-1 + 0.5. A: Analyzer; P: Polarizer.

Figure 2. Z. L. Wu, et al. 

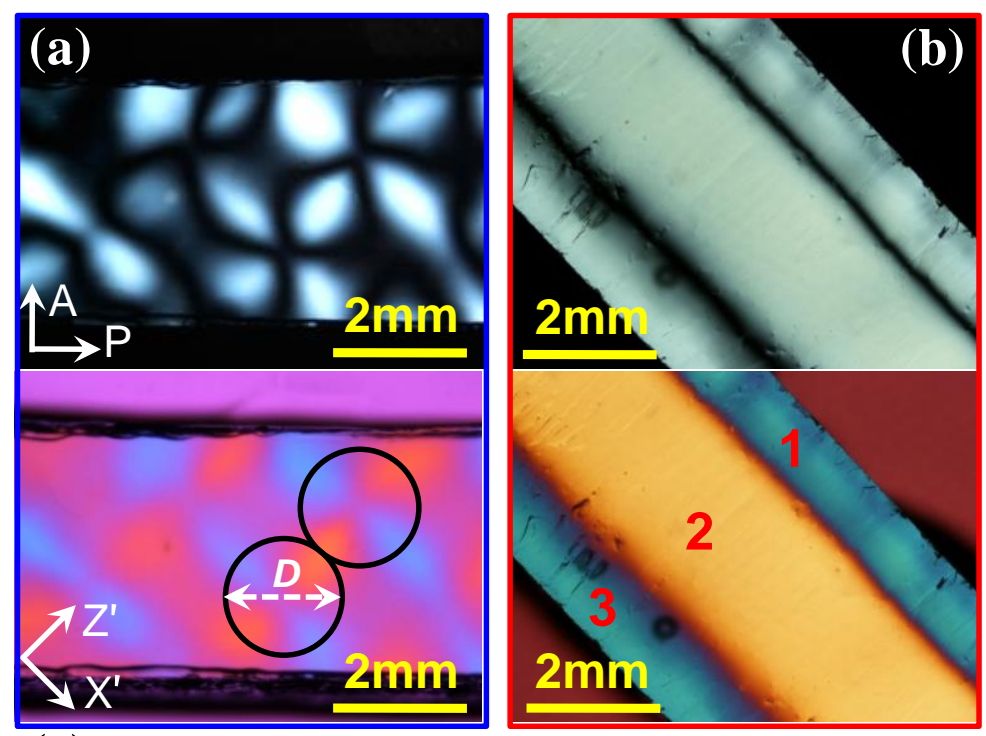

(c)

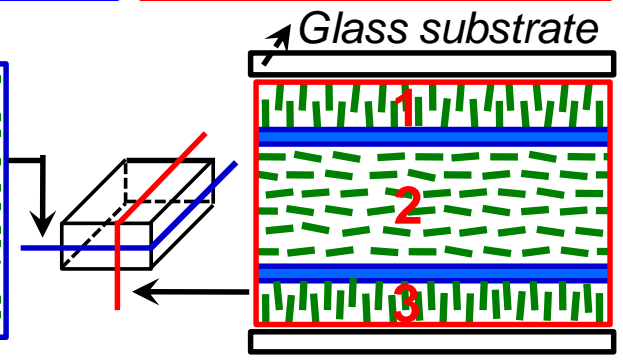

Figure 3. (a,b) Optical polarizing micrographs of the swollen gel QP-1.5-1 + 0.5 observed from the top (a) and the side (b); the upper and lower images are observed without and with $530 \mathrm{~nm}$ tint plate, respectively. The diameter $D$ of the concentric domains is shown in the lower image of (a); three regions $(1,2,3)$ of which the molecules are vertically orientated with each other $(1 \perp 2,2 \perp 3)$ are shown in the lower image of (b). (c) A simple illustration of molecular orientation of PBDT and its semi-rigid polyion complex in gel. $X^{\prime}$ : Fast axis of the tint plate; Z': Slow axis of the tint plate.

Figure 3. Z. L. Wu et al. 
(a)

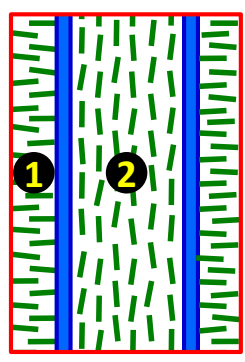

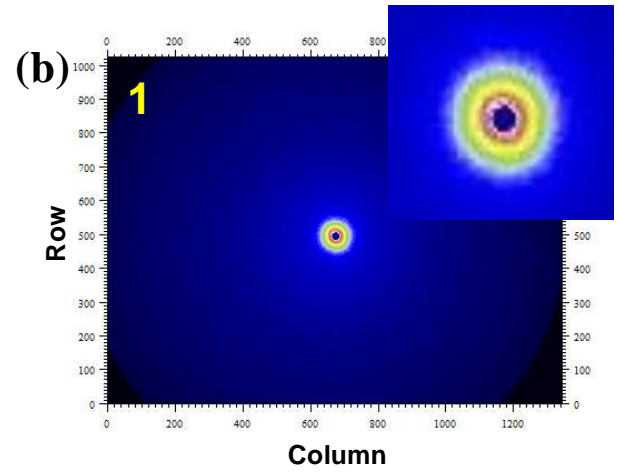

(c)

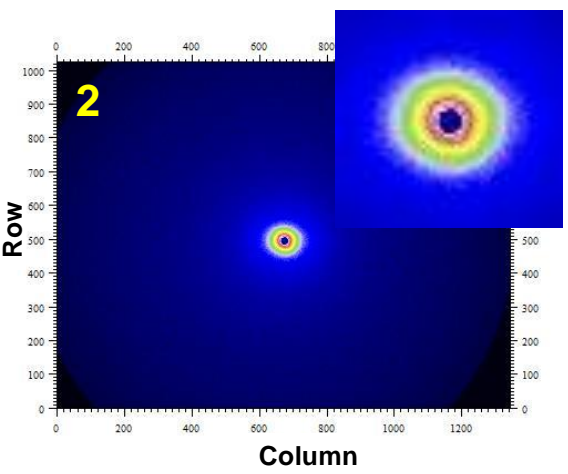

Figure 4. SAXS measurement of the gel QP-1.5-1 + 0.5 from lateral direction. (a) Scheme for the side view of molecular orientation in gel and the relative scattering positions, 1 and 2. (b, c) Scattering patterns of position 1: (b), and 2: (c). The insert images show the enlarged scattering patterns. The weak meridional (b) and equatorial (c) anisotropic scattering patterns indicate the different preferential molecular alignments, as shown in (a).

Figure 4. Z. L. Wu, et al. 


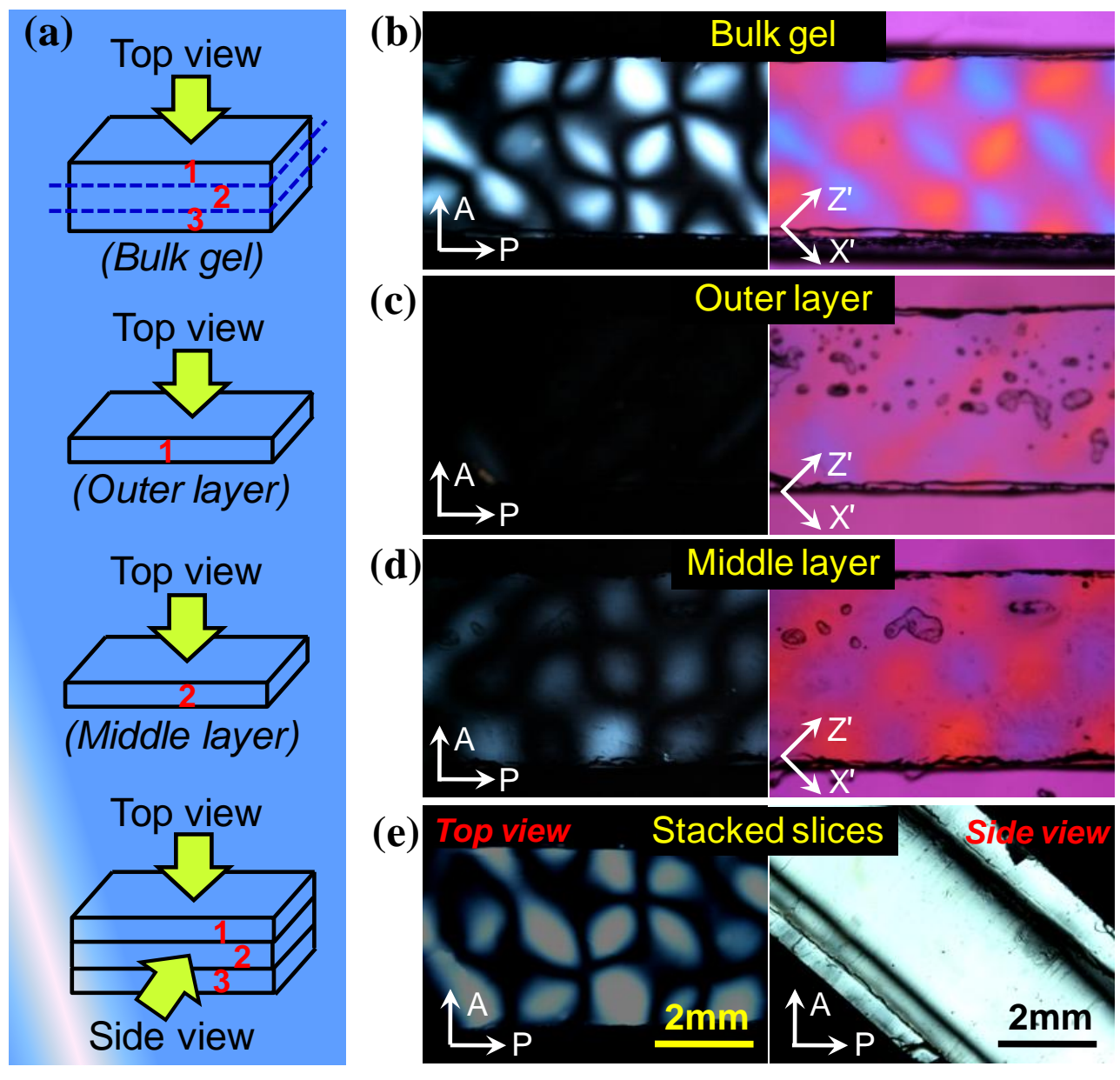

Figure 5. (a) Scheme for the observation of the gel QP-1.5-1 + 0.5 after being cut into slices. (b-e) Micrographs of the bulk and slice gels observed under polarizing microscope; all the images are shown with the same magnification as (e). The bulk gel (b) and sliced gel (c, d) were observed from the top; the right images are observed with $530 \mathrm{~nm}$ tint plate. The stacked gel slices (e) were observed from the top and side.

Figure 5. Z. L. Wu, et al. 
(a)

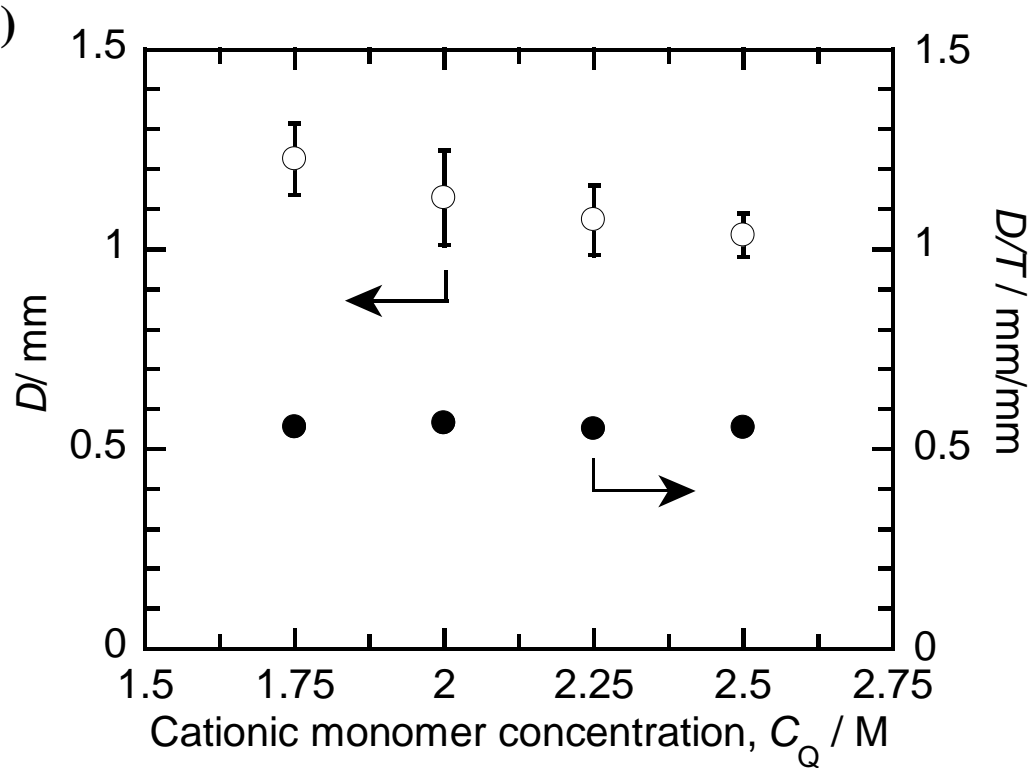

(b)

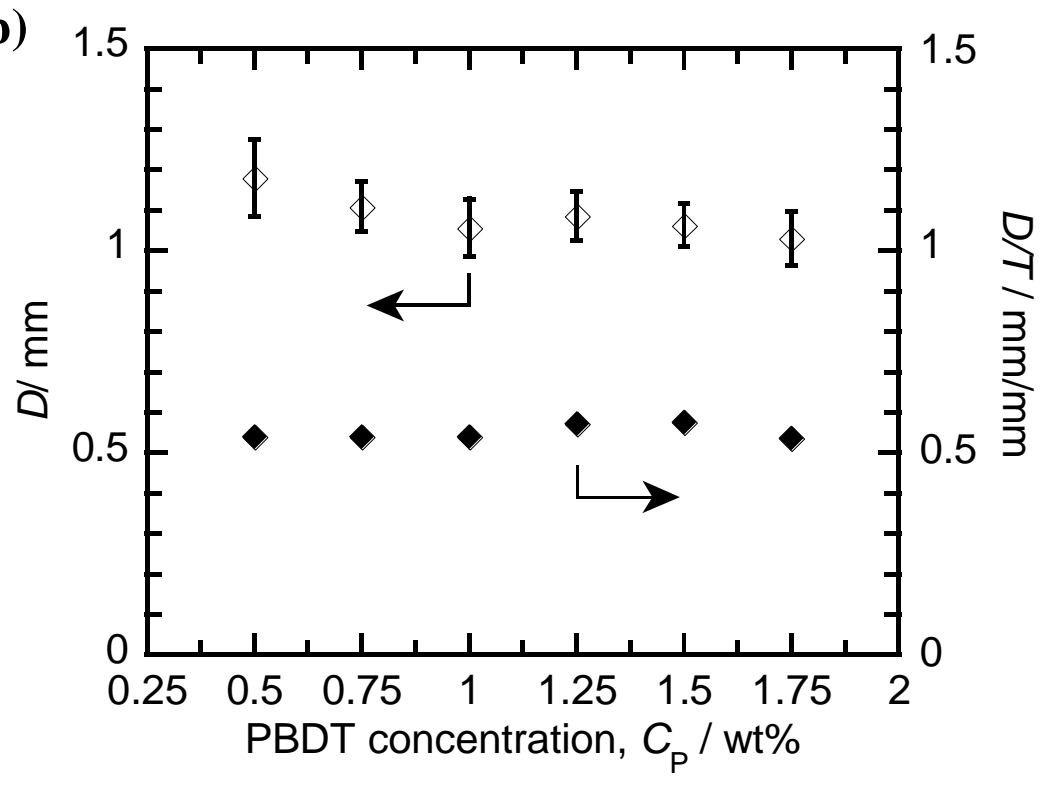

Figure 6. Dependence of the diameter of concentric cylinder $D$ formed in the swollen gel on the composition of the precursor polymerization solution, (a) $\mathrm{QP}-C_{\mathrm{Q}^{-}} 1$; (b) QP-2- $C_{\mathrm{P}}$. The ratio of $D$ to the thickness of the swollen gel $T, D / T$, is shown in the right vertical axis. The gels are synthesized with $1 \mathrm{~mm}$ thickness of silicone spacer.

Figure 6. Z. L. Wu et al. 


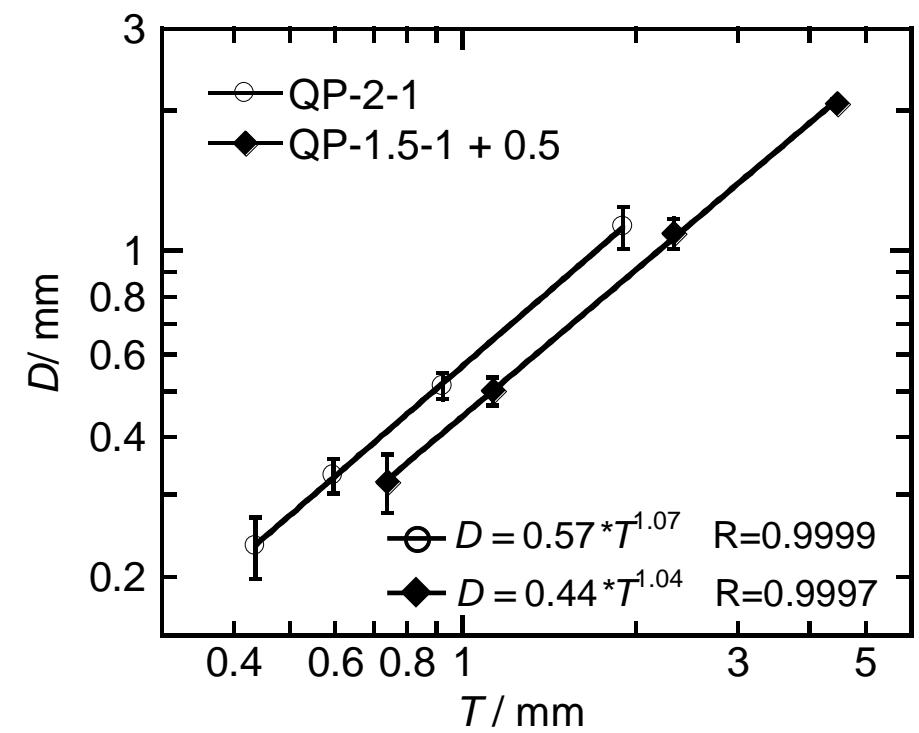

Figure 7. Dependence of the diameter of concentric cylinder $D$ formed in the swollen gel on the swollen gel thickness $T$. Gels were synthesized from the precursor solutions of QP-1.5-1 + 0.5 and QP-2-1 with various thickness of the silicone spacer.

Figure 7. Z. L. Wu et al. 


\section{Table of contents}

Hydrogel with Cubic-Packed Giant Concentric Domains of Semi-Rigid Polyion

\section{Complex}

Zi Liang Wu, Md. Arifuzzaman, Takayuki Kurokawa, Hidemitsu Furukawa and Jian Ping Gong*

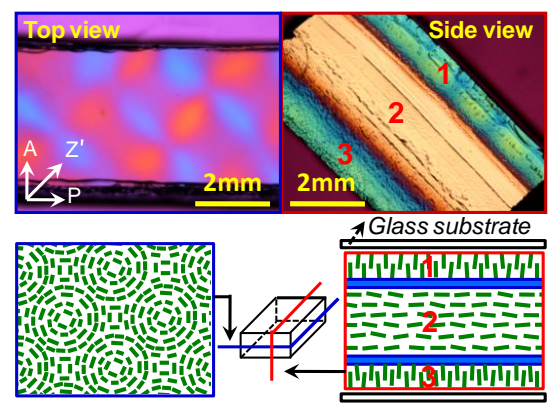

The giant structure in hydrogel, formed via self-assembly of a semi-rigid polyion complex, consists of millimeter-scale concentric domains that are cubically packed in the inner region of the hydrogel and sandwiched by two homeotropically aligned surface layers. 